\title{
Guest Editorial for the Special Issue on Antennas and Propagation at mm- and Sub mm-Waves
}

M ILLIMETER and sub-millimeter waves have been attracting intensive research for quite some time. It has extended from high-cost niche applications, mostly related to military, radio astronomy and space applications, to embrace widespread new consumer applications, some of which with strong potential for mass market. The opportunity for these bands is in part created by the spectrum availability, high spectral capacity to offer extremely high data rates or high resolution, and the inherent potential for miniaturization, lightweight and low-cost of the front-end. Since the previous IEEE TRANSACTIONS ON ANTENNAS AND PROPAGATION Special Issue on Antennas and Propagation Aspects of $60-90 \mathrm{GHz}$ Wireless Communications in 2011, we have seen a big spike in the $\mathrm{mm}$ - and sub-millimeter wave antenna research in part due to fast parallel advancements on the supporting technology (affordable compact sources, inexpensive silicon-based integrated circuit electronics, micro-fabrication technologies) and due to the strong drive from consumer applications in the area of personal communications, security and automotive safety.

However challenges still remain for antennas in package, antennas on chip, phased arrays, focusing antennas, radiowave propagation modeling as well as for new devices that bridge between traditional antenna topics and other interdisciplinary areas. The desirable trend for front-end miniaturization and system integration on-package or on-chip raises the challenge for antenna integration without sacrificing its radiation efficiency, bandwidth and achievable gain. Some consumer market wireless applications require advanced beamforming or very high gain steerable beams to maintain the link. This usually requires larger arrays or larger radiating apertures, but again high precision fabrication techniques are required, as well as error tolerant designs, low loss interconnections and precise evaluation of material electrical characteristics. Typical consumer market applications are short range indoor or outdoor and recently body area application have brought the range to some tens of centimeters. In all cases adequate broadband channel models are required to enable system level design. At these frequencies diffraction losses are excessively high, but multipath richness may be higher favoring for instance MIMO strategies. The field of nano-optics has also recently emerged, boosted by the development of nanoscience and nanotechnology, covering topics such as near-field optics, single-molecule microscopy, nano-antennas, plasmonics and metamaterials. Recent progress in nanofabrication techniques has also induced an increasing interest in the scattering and emission properties of nanoparticles and confined optical sources, building a first bridge between physics and engineering communities.

Digital Object Identifier 10.1109/TAP.2013.2248491

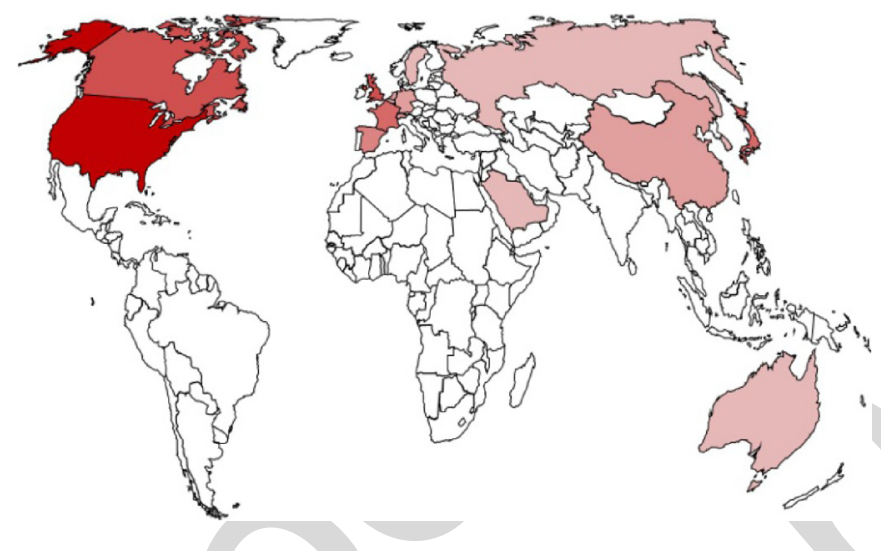

Fig. 1. Countries of origin of the published papers. Darker color represents higher number of papers.

The objective of this special issue is to provide an overview of recent advances and emerging applications in millimeter and sub-millimeter waves, especially beyond $60 \mathrm{GHz}$ up to terahertz, with strong focus towards practical implementable solutions. From a total of 93 submissions, 43 papers were selected, which present remarkable advancements to the state of the art and which are especially stimulating for the Antennas and Propagation community. The papers originate from a broad list of research centers around the world (see Fig. 1) and cover topics in the area of antennas, from mm-waves to optical wavelengths, $\mathrm{mm}$-wave devices and propagation modeling.

We hope that this special issue may serve as a reference for researchers developing the next generation of $\mathrm{mm}$ - and sub $\mathrm{mm}$-wave antennas and wireless systems.

\section{Contents of the Special Issue}

This issue is divided into five main sections (Fig. 2.). About one third of the accepted papers deal with focusing or collimating antennas (planar and volumetric antenna solutions). Compact antennas and antennas in package, mostly in the 60 $\mathrm{GHz}$ band, are described in twelve papers. Antenna arrays and array theory represent $19 \%$ of the total number of accepted papers, and four papers deal with channel modeling and characterization. It is also interesting to note that a few papers focus on nanostructures and other $\mathrm{THz}$ devices.

\section{A. Nanostructures and Other THz and mm-Wave Devices (5 Papers)}

Advances in nanofabrication have opened an exciting new area of electromagnetic study related to the interaction between the nanoscale details of materials and light. Optical antennas are one such example that bridge between nanoscale optical sources, light-matter interaction and far-field radiation. New 


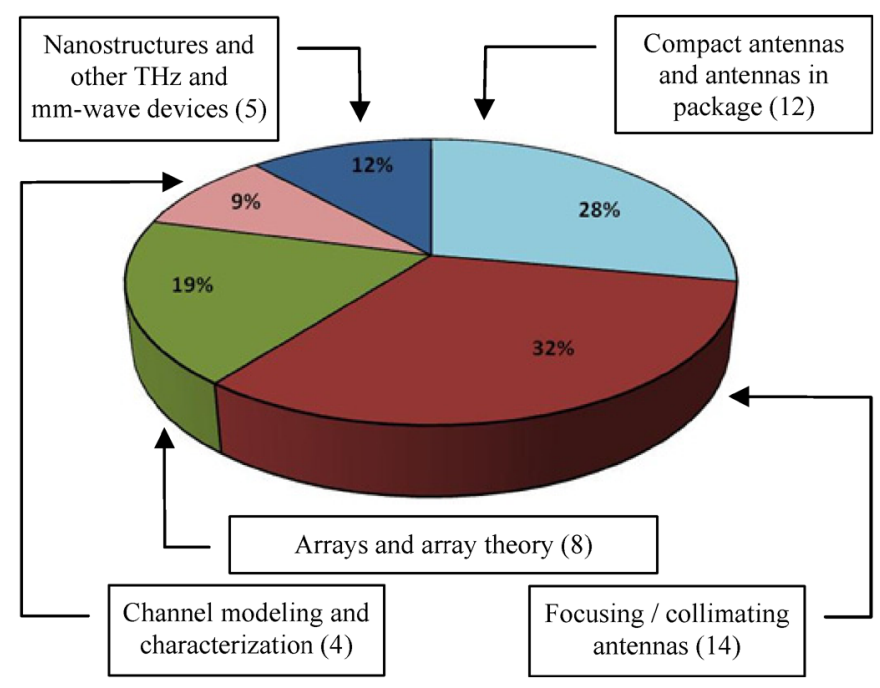

Fig. 2. Sections of the special issue. Numbers in parentheses are the number of papers in each section.

solar cell solutions with nanostructured surface are another example. Material properties and wave-matter interaction change near infrared and optical wavelengths in a way that does not allow direct translation of established design concepts to these frequencies. The special issue opens with an invited paper by Alù et al. that presents an in depth review of some of the concepts of optical antenna theory, design and applications. In another paper, Brockett et al. present a detailed electromagnetic characterization of GaAs photovoltaic nanopillar array solar cells for solar energy harvesting. The numerical results show significant increase in solar power absorbance over that of conventional bulk solar cells.

Phase shifters are an essential device in a phased array to allow for beam steering. At $\mathrm{mm}$ and sub mm-waves those devices are expensive, complex and sometimes unreliable. Chen et al. present the conceptual vision of phase shifters based on integrally-gated graphene parallel-plate waveguides for operation at $\mathrm{THz}$ and infra-red. With this type of technology it might be possible in the future to realize low-loss and extremely compact on-chip programmable phasing devices.

Enhancement of $\mathrm{THz}$ wave generation efficiency is another continuous challenge. Khiabani et al. analyze the factors affecting the radiated power and optical-THz power conversion efficiency of a $\mathrm{THz}$ photoconductive antenna, using an equivalent circuit lumped-elements model.

Space borne calibration targets are currently a common practice for in-flight passive radiometer calibration for remote sensing applications. Measurement reliability depends on the accuracy of the load, which is influenced by its reflectivity level across the band and temperature uniformity over the device. Design constraints are its mass and volume. Murk et al. present a new load configuration that offers a good compromise between radiometric performance and the design constrains. Radiometric performance is evaluated by simulation and measurements on a breadboard model. Thermal behavior of the structure is studied by simulation.

\section{B. Compact Antennas and Antennas in Package (12 Papers)}

There is an increasing demand for low-cost wireless communication and imaging systems that operate in the mm-wave bands. Recent advances in silicon technologies have enabled single chip solutions, resulting in substantial cost reductions. However, to flourish the market of these mm-wave systems, not only are low-cost radio frequency integrated circuit (RFIC) solutions required, but also low-cost RFIC packages with integrated antennas. A paper by Tsutsumi et al. presents an antenna in package solution for $60 \mathrm{GHz}$ in which a loop antenna consists of two bonding wires connecting to a complementary metal-oxide-semiconductor (CMOS) chip and a metal plate on an interposer in a ball grid array (BGA) package. The antenna can be fabricated at low cost adopting a conventional BGA package fabrication process. The paper by Beer et al. proposes a packaging solution for the integration of a monolithic microwave integrated circuit (MMIC) and a thin film antenna into a single surface-mountable package. As the antenna is placed above an air cavity, a large bandwidth is achieved for the antenna. An ultimate low cost V-band solution comes from a paper by Hong et al. In this paper, the antenna is implemented on the application board directly, thus eliminating a traditional antenna package and its complicated grid array assemblies.

Most antenna-in-package designs use planar structures, such as patch, dipole, loop or slot. The paper by Enayati et al. proposes a horn-type structure at $60 \mathrm{GHz}$. The design yields widebandwidth and is suitable for array applications where nonline-of-sight communication is needed or multi-path spatial diversity techniques are to be implemented. A bond-wire-free interconnection between a MMIC and an E-band antenna using a stacked patch configuration is investigated by Smith et al.A short horn has been used to increase the antenna directivity. Alternatively, Ohlsson et al. present a slot-coupled semiconductor dielectric resonator antenna designed for on-chip assembly. The antenna has an impedance bandwidth of $6.1 \%$ in the V-band and a radiation efficiency of $98 \%$.

There are many papers discussing on-chip antenna designs. On-chip antennas typically have either very low radiation efficiency or very narrow impedance bandwidth due to the $\mu \mathrm{m}$-range distance between the planar antenna structure and the ground plane in conventional complementary metal-oxide-semiconductor (CMOS) or SiGe processes and the lossy silicon used (resistivity typically in the $1-10 \Omega . c m$ range). The paper by Marnat et al. proposes a new movable plate concept to realize mm-wave vertical on-chip antennas through microelectromechanical systems (MEMS) based post-processing steps in a CMOS compatible process. By virtue of its vertical position, the antenna is isolated from the lossy Si substrate and hence performs with a better efficiency as compared to the horizontal position. In addition, the movable plate concept enables polarization diversity by providing both horizontal and vertical polarizations on the same chip.

Most of the proposed $60 \mathrm{GHz}$ antennas in package present a boresight radiation pattern. However for some applications it is actually desirable to have an end-fire antenna. A wide-angle radiation pattern fan-like printed antenna designed for wireless communications in the V-band is proposed by Sun et al. 
A different application scenario is addressed in the article by Yeh et al., namely chip-to-chip communications. Wireless communications in principle can reduce the crowdedness and even improve system performance associated with wired connections. Comparing to a regular patch antenna, the patch antenna with an artificial magnetic conductor layer provides not only gain improvement in the horizontal direction but also very wide impedance bandwidth.

Other compact but non-packaged antenna solutions are also included in this section. Developing wideband architectures with multiple-input-multiple-output (MIMO) antenna systems at mm-wave offer many advantages including jointly optimized analogue and digital signal processing. This allows for flexible antenna designs and insertion loss reduction, as many passive structures can be avoided at both sides of the link. The paper by Ariza et al. presents a $60 \mathrm{GHz}$ polarimetric MIMO sensing system architecture that includes analogue miniaturized front ends. This architecture permits compact MIMO radar designs, multi-dimensional channel sounding for multi-gigabit communications, and the study of indoor polarimetric wireless channels in static and shadowed conditions due to human activity. In another paper, Bhattacharyya et al. propose a new analysis method for ring-slot elements backed by ground plane via cavities performed using a hybrid matrix formulation. The validity of the method has been demonstrated against finite element method (FEM) simulations while the new formulation is at least one order of magnitude faster. An optically transparent $\mathrm{CPW}$-fed monopole lozenge antenna using silver grid layer is described by Hautcoeur et al. for $60 \mathrm{GHz}$ security applications.

\section{Focusing/Collimating Antennas (14 Papers)}

Antennas for $\mathrm{mm}$ and sub mm-wave applications typically require high gain to compensate for high free-space propagation loss. Renewed interest is for indoor multi-gigabit links or for automotive road safety applications. At these frequencies lenses are an attractive solution either as a primary feed or as a standalone moderate gain antenna. Nguyen et al. present a shaped dome V-band dielectric lens with stable Gaussian-like radiation pattern over a $25 \mathrm{GHz}$ bandwidth. This configuration presents advantage of lower material losses and lower weight compared to conventional lens solutions. Dielectric lenses can be used as array elements for beam steering as proposed by Artemenko et $a l$. This requires fewer array elements than a phased array for the same gain and allows replacing expensive phase shifters by RF switches.

In some application like automotive radars, a narrow beam is required only in one plane while in the orthogonal plane the beam can be broader. In this case instead of a 3D lens, a flat lens can be used. Lafond et al. present a flat Luneburg lens with reconfigurable feeds using individual MMIC amplifier and single pole, single throw (SPST) switch at each feed branch for very wide-angle electronic beam scanning. The paper demonstrates the feasibility of the concept at the $60 \mathrm{GHz}$ band.

Fresnel zone plates (FZP) can be used as an alternative to 3D dielectric lenses when large bandwidths are not required. The FZP antennas feature the merits of planar surface, reduced height and weight, when compared with the conventional dielectric lenses. A compact cavity-backed FZP lens antenna is presented by Xu et al. The antenna presents a boresight gain of $20.8 \mathrm{dBi}$ and a $3 \mathrm{~dB}$ gain bandwidth from 266 to $275 \mathrm{GHz}$. The back cavity improves aperture illumination, suppresses back radiation and reduces spillover losses. In a similar topic, Gallacher et al. introduce the design and optimization of a photo-injected FZP antenna. In this type of antennas the transmission loss of the different confined regions on the FZP plasma can be optically controlled. This way it is possible to dynamically focus and/or steer a directive beam at $\mathrm{mm}$ - or sub mm-waves.

Reflector antennas may be more favorable when much higher gains are required. Unlike lenses, the aperture size can be increased without increase of losses. A short bowtie antenna with a broadband impedance bandwidth from 56 to $100 \mathrm{GHz}$ is presented by $\mathrm{Qu}$ et al. The gain is increased up to $18.5 \mathrm{dBi}$ by placing the bowtie in the focus of a small elliptically shaped metallic cavity.

Reflectarray is a type of reflector than can be made reconfigurable by dynamic altering the properties of each unit-cell. Compared to conventional phased arrays, reconfigurable reflectarrays tend to have lower cost and ease of construction. The paper by Perez-Palomino et al. presents a more flexible reconfigurable reflectarray concept obtained by printing it above a liquid crystal. The model takes into account the anisotropy and inhomogeneity of the liquid crystal. A demonstrating prototype is designed and measured in the F-band.

A particular challenge for the design of a reflector system at $\mathrm{mm}$ - and sub $\mathrm{mm}$-waves is the design of feasible feeding elements. The methodology and tools for the design of short, ultra-low side-lobe corrugated feed horns in the $\mathrm{mm}$ - and the sub mm-wave bands is presented by McKay et al. Furthermore, the challenge increases when high density of feeds is required in the reflector focal plane to improve spatial information, as happens with imaging applications. Renker et al. analyze the focal plane array for a Stratosphere-Troposphere radiometer, while Trichopoulos et al.introduce a focal plane array sensor for $\mathrm{THz}$ imaging in the $0.6-1.2 \mathrm{THz}$ band. Each pixel in the sensor array consists of broadband $\mathrm{THz}$ antennas monolithically integrated with ultra-fast heterostructure backward diodes for $\mathrm{THz}$ sensing.

Sun et al. present a gain enhancement method for an antipodal tapered slot antenna by using zero-index metamaterial in the open aperture region of the antenna. Adding the zero-index metamaterial does not affect the antenna impedance much and allows a gain of $10.6-13.1 \mathrm{dBi}$ over $60 \mathrm{GHz}$ band. The paper by Ghassemi and $\mathrm{Wu}$ describes another gain enhancement method by dielectric-loading the antenna in the aperture area. Compared to the zero-index metamaterial method, this one provides more gain improvement and maintains a much wider bandwidth. In a similar topic, Taringou et al. present a long broadband antipodal tapered slot antenna fed by a coplanar waveguide (CPW) instead of the more common microstrip feeding. CPW technology has some advantage over microstrip lines which include surface-mount integration, lower phase velocity variation, lower cross talk and radiation. 
Determination of the far-field radiation pattern of a high gain antenna using the classical near-filed to far-field transformation at $\mathrm{mm}$ - and sub mm-waves may be hindered by the difficulties to accurately retrieve phase. Junkin describes a new technique for accurate phase retrieval from the far-fields radiated by a Terahertz antenna from measured near-field intensity. Authors use graphics processing units (GPUs) to obtain almost real-time accurate far-field calculations.

\section{Arrays and Array Theory (8 Papers)}

Arrays are an alternative to focusing antennas when high gain is required but low profile is mandatory, for example, to allow package level integration. Due to its high manufacturing precision and capabilities of implementing complicated via structures, low temperature co-fired ceramics (LTCC) technology is a promising candidate for mm-wave antenna arrays. One major issue with mm-wave planar arrays is the relative strong surface wave due to large substrate size, particularly with high dielectric constant LTCC substrates. There are two major methods to reduce surface waves. One is to use low dielectric substrate such as foam materials or air cavity in the design. But air cavity increases manufacturing difficulties and cost while foam is very limited for mm-wave applications. The other method is to use electromagnetic band-gap (EBG) or ring structures around the antenna radiator to reduce surface waves. But it is difficult to use these concepts in array designs. The paper by Wang et al. proposes to use soft-surface structures comprised of metal strips and via fences to improve the radiation performance by reducing the losses due to severe surface waves and mutual coupling between adjacent elements. She et al. describe an oversized rectangular waveguide slot array antenna in V-band with an air layer in the radiating part to enhance the antenna gain. The array is fabricated using LTCC. The insertion of the air layer allows for a reduction on dielectric loss and bandwidth enhancement. In a similar topic, $X u$ et al. present a broadband and high gain array operating at $140 \mathrm{GHz}$ using dielectric-loaded slots fed by a substrate integrated waveguide (SIW). The configuration is electrically robust and has a simple design for fabrication using LTCC technology. In the $270 \mathrm{GHz}$ band, the previous authors now introduce a planar high gain linearly polarized radial line slot array antenna manufactured in LTCC technology.

Sano et al. present a linear slot array in a hollow rectangular coaxial waveguide in the V-band. This configuration allows fabrication using a different technology: diffusion bonding of laminated thin metal plates. A particularly interesting solution is presented to support the inner conductor in the center of the hollow waveguide. A totally different array technology and support material is discussed in Chahat et al. paper, It demonstrates a microstrip patch antenna array printed on commercial textiles at mm-waves for wearable applications and proposes a new fabrication process for reliable and accurate manufacturing of mm-wave microstrip antennas on textiles.

This section includes also new array concept developments. A possible array configuration to obviate the need for precise phase shifter is presented by Krieger et al. The proposed architecture uses arrays with elements spaced more closely than otherwise required over the target aperture in conjunction with novel spatial-domain delta-sigma processing to enable high performance with low-resolution phase shifters.

A two-dimensional array is presented by Lemaitre-Auger et $a l$. as a solution to generate a collimated and confined Bessel beam. The authors investigate both the array lattice and the effect of sub-sampling the array in order to reduce the number of antenna elements.

\section{E. Channel Modeling and Characterization (4 Papers)}

Indoor and outdoor wireless fixed and mobile communication services are steadily demanding ever increasing capacity to support huge traffic growth generated by increasing number of users and increasing number of mobile user broadband platforms and applications. Required bandwidth for next generation cellular networks is available at the under-utilized millimeter-wave spectrum. Adequate channel characterization is required at these frequencies in the context of cellular communications, using high gain beam-steerable antennas. Rappaport et al. present an experimental study on mm-wave propagation for outdoor cellular communication systems in urban environment, considering high gain beam steering antennas. The paper presents measurement results for the $40 \mathrm{GHz}$ band and propagation models relevant for future planning and deployment of such mobile systems. With a similar motivation, García-Rubia et al. analyze the results from a two-year rain attenuation measurement campaign at $80 \mathrm{GHz}$ over a horizontal $840 \mathrm{~m}$ link at Madrid. Measured attenuation results are compared with established theoretical models using drop-size distribution functions obtained from parallel experiments carried out at the same location.

Multi gigabit networks are being studied for indoor scenarios at millimeter waves. As for outdoor scenarios, high gain antennas are required to ensure the link budget, but obstruction by the human body may impair the service. Takahashi et al. propose a solution to minimize communication interruption at 60 $\mathrm{GHz}$ band by the human body by using artificial multi reflectors. The improvements by the proposed method are quantified; the strategy is generally applicable to most indoor communications environments.

Very high data-rates are also required for emerging body-area network applications. Again millimeter-waves are a potential candidate to accommodate the required large bandwidths and so channel model characterization is needed for this very specific type of scenarios. Chahat et al. characterize the electromagnetic fields propagating at the skin/air interface at $60 \mathrm{GHz}$ for on-body networks. The study comprises analytical formulation, full-wave simulation and experiments. The main focus is on the interpretation, in the near-skin context, of the different terms from the old Norton approximate expressions derived from Sommerfled's formulation for a dipole near a lossy medium. The interest of the paper is put also on the determination of the power loss exponent in different regions.

\section{ACKNOWLEDGMENT}

The Guest Editors want to express their sincere thanks to Prof. Michael A. Jensen, Editor-in-Chief of the IEEE TRANSACTIONS ON ANTENNAS AND PROPAGATION, for supporting, encouraging 
and overseeing in the elaboration /I think you mean "collaboration" and not "elaboration"] and

publication of this special issue. They thank all the authors for their outstanding contributions and the more than 200 reviewers who were involved in the reviewing process of more than 90 submissions. As we all know, the quality of the published articles highly depends on the authors and also on the work done by the reviewers with their useful suggestions and comments that helped improving the overall quality of the papers. To all, thank you.
CARlos FERnANDES FERNANDES Guest Editor
Instituto de Telecomunicações, Instituto Superior Técnico
Technical University of Lisbon
Lisbon, Portugal

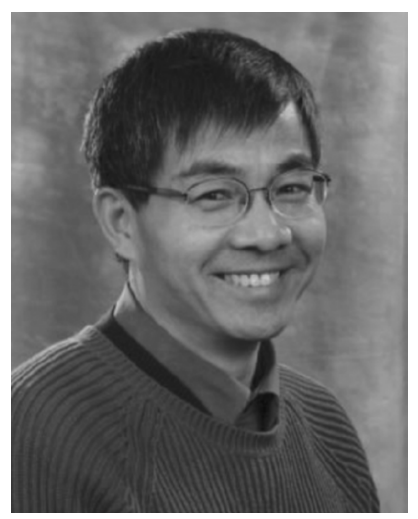

Duixian Liu (S'85-M'90-SM'98-F'10) received the B.S. degree in electrical engineering from XiDian University, Xi'an, China, in 1982, and the M.S. and Ph.D. degrees in electrical engineering from the Ohio State University, Columbus, OH, USA, in 1986 and 1990, respectively.

From 1990 to 1996, he was with Valor Enterprises Inc., Piqua, OH, USA, initially as an Electrical Engineer and then as the Chief Engineer, during which time he designed an antenna product line ranging from $3 \mathrm{MHz}$ to $2.4 \mathrm{GHz}$ for the company, a very important factor for the prestigious Presidential "E" Award for Excellence in Exporting in 1994. Since April 1996, he has been with the IBM T. J. Watson Research Center, Yorktown Heights, NY, USA, as a Research Staff Member. He has received three IBM's Outstanding Technical Achievement Awards and one Corporate Award, the IBM's highest technical award. He was named Master Inventor in 2007. He has co-edited a book titled Advanced Millimeter-wave Technologies-Antennas, Packaging and Circuits (Wiley, 2009). He has authored or coauthored more than 100 journal and conference papers.

Dr. Liu is a Fellow of IEEE. He received the Best Paper Prize of the 2007 IEEE International Workshop on Antenna technology for “Antenna-in-package in LTCC for 60 GHz Radio," IBM Research's 2011 Pat Goldberg Memorial Best Paper Award in Computer Science, Electrical Engineering and Math for "Organic Packages with Embedded PhasedArray Antennas for 60-GHz Wireless Chipsets," and the 2012 S. A. Schelkunoff Prize Paper Award of the IEEE Antennas and Propagation Society for "Dual Grid Array Antennas in a Thin-Profile Package for Flip-Chip Interconnection to Highly Integrated 60-GHz Radioß? He has 40 patents issued and 20 patents pending. His research interests are antenna design, EM modeling, chip packaging, digital signal processing, and communications technologies. He is an Associate Editor for the IEEE TRANSACTIONS ON ANTENNAS AND Propagation and was a Guest Editor for the July 2009 IEEE TRANSACTIONS ON ANTENNAS AND PROPAGATION Special Issue on Antennas and Propagation Aspects of 60-90 GHz Wireless Communications. He has been the Organizer or Chair for numerous international conference sessions or special sessions and also a technical program committee member. He was the General Chair of the 2006 IEEE International Workshop on Antenna Technology: Small Antennas and Novel Metamaterials, White Plains, NY. He has served as an external Ph.D. examiner for several universities and external examiner for government organizations on research grants.

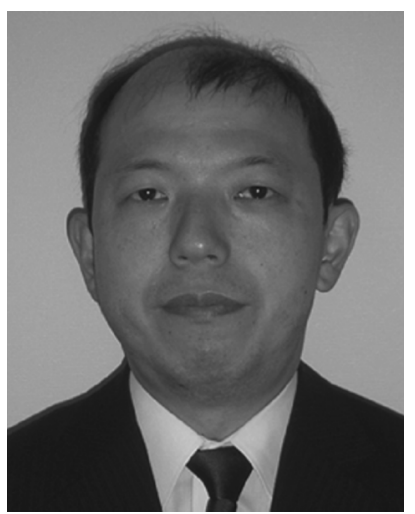

Jiro Hirokawa (S'89-M'90-SM'03-F'12) was born in Tokyo, Japan, on May 8, 1965. He received the B.S., M.S., and D.E. degrees in electrical and electronic engineering from Tokyo Institute of Technology (Tokyo Tech), Tokyo, Japan in 1988, 1990 and 1994, respectively.

He was a Research Associate from 1990 to 1996, and is currently an Associate Professor at Tokyo Tech. From 1994 to 1995, he was with the antenna group of Chalmers University of Technology, Gothenburg, Sweden, as a Postdoctoral Fellow. His research area has been in slotted waveguide array antennas and millimeter-wave antennas.

Prof. Hirokawa is an IEEE Fellow and a Senior Member of IEICE. He received an IEEE AP-S Tokyo Chapter Young Engineer Award in 1991, a Young Engineer Award from IEICE in 1996, a Tokyo Tech Award for Challenging Research in 2003, a Young Scientists' Prize from the Minister of Education, Cultures, Sports, Science and Technology in Japan in 2005, a Best Paper Award in 2007, a Best Letter Award in 2009 from IEICE Communications Society and Asia Pacific Microwave Conference prize in 2011 and 2012. 

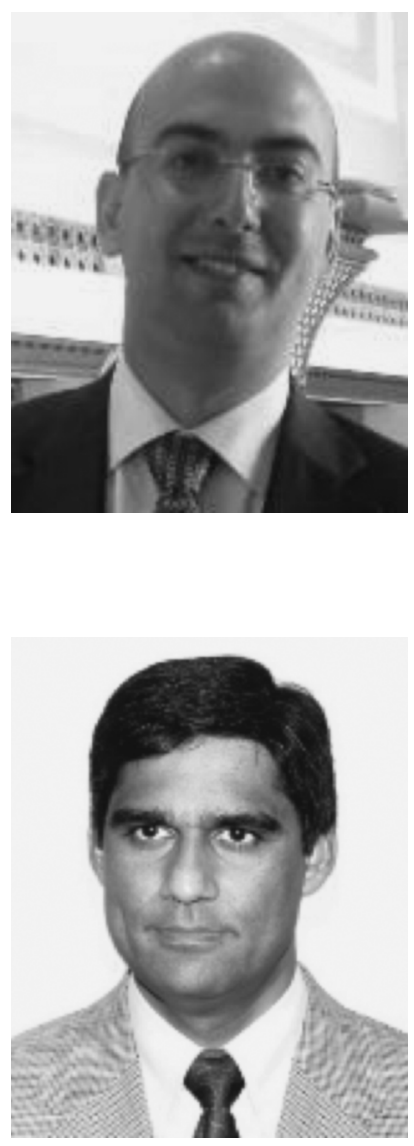

and metamaterials.

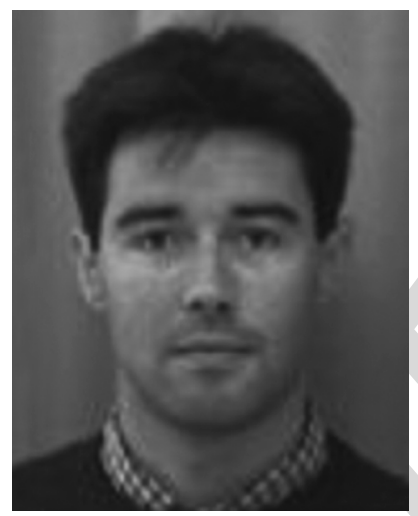

Ronan Sauleau (M'04-SM'06) received the B.Sc. degree in electrical engineering and radio communications from the Institut National des Sciences Appliquées, Rennes, France, in 1995. He received the Agrégation degree from the Ecole Normale Supérieure de Cachan, Cachan, France, in 1996, and the Ph.D. degree in signal processing and telecommunications and the "Habilitation à Diriger des Recherche" degree from the University of Rennes 1, Rennes, France, in 1999 and 2005, respectively.

He was an Assistant Professor and Associate Professor at the University of Rennes 1, Rennes, France between September 2000 and November 2005, and between December 2005 and October 2009, respectively. He has been appointed as a Full Professor in the same University since November 2009. His current research fields are numerical modeling (mainly FDTD), millimeter-wave printed and reconfigurable (MEMS) antennas, substrate integrated waveguide antennas, lens-based focusing devices, periodic and nonperiodic structures (electromagnetic bandgap materials, metamaterials, reflectarrays, and transmitarrays), and biological effects of millimeter waves. He has been involved in more than 30 research projects at the national and European levels and has co-supervised 14 Postdoctoral Fellows, 24 Ph.D. students, and 40 Master students. He has received eight patents and is the author or coauthor of 135 journal papers and more than 285 publications in international conferences and workshops. He has shared the responsibility of the research activities on antennas at IETR in 2010 and 2011. He is now co-responsible for the research Department "Antenna and Microwave Devices" at IETR and is deputy director of IETR.

Prof. Sauleau received the 2004 ISAP Conference Young Researcher Scientist Fellowship (Japan) and the first Young Researcher Prize in Brittany, France, in 2001, for his research work on gain-enhanced Fabry-Perot antennas. In September 2007, he was elevated to junior member of the "Institut Universitaire de France". He was awarded the Bronze medal by CNRS in 2008. He was the co-recipient of several international conference awards (Int. Sch. of BioEM 2005, BEMS'2006, MRRS'2008, E-MRS'2011, BEMS'2011, IMS'2012, Antem'2012). 


\section{Guest Editorial for the Special Issue on Antennas and Propagation at $\mathrm{mm}$ - and Sub $\mathrm{mm}$-Waves}

M ILLIMETER and sub-millimeter waves have been attracting intensive research for quite some time. It has extended from high-cost niche applications, mostly related to military, radio astronomy and space applications, to embrace widespread new consumer applications, some of which with strong potential for mass market. The opportunity for these bands is in part created by the spectrum availability, high spectral capacity to offer extremely high data rates or high resolution, and the inherent potential for miniaturization, lightweight and low-cost of the front-end. Since the previous IEEE Transactions ON ANTENNAS AND Propagation Special Issue on Antennas and Propagation Aspects of $60-90 \mathrm{GHz}$ Wireless Communications in 2011, we have seen a big spike in the mm- and sub-millimeter wave antenna research in part due to fast parallel advancements on the supporting technology (affordable compact sources, inexpensive silicon-based integrated circuit electronics, micro-fabrication technologies) and due to the strong drive from consumer applications in the area of personal communications, security and automotive safety.

However challenges still remain for antennas in package, antennas on chip, phased arrays, focusing antennas, radiowave propagation modeling as well as for new devices that bridge between traditional antenna topics and other interdisciplinary areas. The desirable trend for front-end miniaturization and system integration on-package or on-chip raises the challenge for antenna integration without sacrificing its radiation efficiency, bandwidth and achievable gain. Some consumer market wireless applications require advanced beamforming or very high gain steerable beams to maintain the link. This usually requires larger arrays or larger radiating apertures, but again high precision fabrication techniques are required, as well as error tolerant designs, low loss interconnections and precise evaluation of material electrical characteristics. Typical consumer market applications are short range indoor or outdoor and recently body area application have brought the range to some tens of centimeters. In all cases adequate broadband channel models are required to enable system level design. At these frequencies diffraction losses are excessively high, but multipath richness may be higher favoring for instance MIMO strategies. The field of nano-optics has also recently emerged, boosted by the development of nanoscience and nanotechnology, covering topics such as near-field optics, single-molecule microscopy, nano-antennas, plasmonics and metamaterials. Recent progress in nanofabrication techniques has also induced an increasing interest in the scattering and emission properties of nanoparticles and confined optical sources, building a first bridge between physics and engineering communities.

Digital Object Identifier 10.1109/TAP.2013.2248491

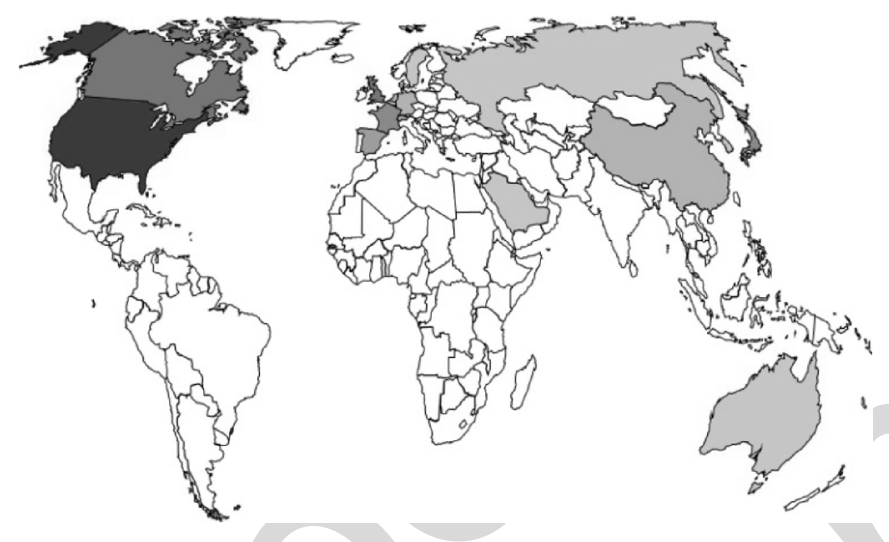

Fig. 1. Countries of origin of the published papers. Darker color represents higher number of papers.

The objective of this special issue is to provide an overview of recent advances and emerging applications in millimeter and sub-millimeter waves, especially beyond $60 \mathrm{GHz}$ up to terahertz, with strong focus towards practical implementable solutions. From a total of 93 submissions, 43 papers were selected, which present remarkable advancements to the state of the art and which are especially stimulating for the Antennas and Propagation community. The papers originate from a broad list of research centers around the world (see Fig. 1) and cover topics in the area of antennas, from mm-waves to optical wavelengths, mm-wave devices and propagation modeling.

We hope that this special issue may serve as a reference for researchers developing the next generation of $\mathrm{mm}$ - and sub $\mathrm{mm}$-wave antennas and wireless systems.

\section{Contents of the Special Issue}

This issue is divided into five main sections (Fig. 2.). About one third of the accepted papers deal with focusing or collimating antennas (planar and volumetric antenna solutions). Compact antennas and antennas in package, mostly in the 60 $\mathrm{GHz}$ band, are described in twelve papers. Antenna arrays and array theory represent $19 \%$ of the total number of accepted papers, and four papers deal with channel modeling and characterization. It is also interesting to note that a few papers focus on nanostructures and other $\mathrm{THz}$ devices.

\section{A. Nanostructures and Other THz and mm-Wave Devices (5 Papers)}

Advances in nanofabrication have opened an exciting new area of electromagnetic study related to the interaction between the nanoscale details of materials and light. Optical antennas are one such example that bridge between nanoscale optical sources, light-matter interaction and far-field radiation. New 


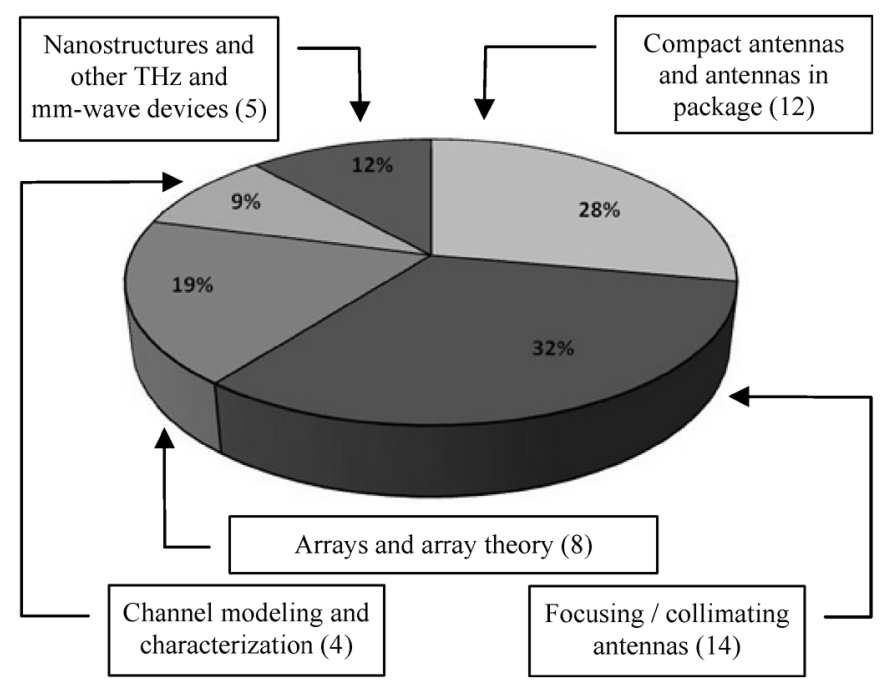

Fig. 2. Sections of the special issue. Numbers in parentheses are the number of papers in each section.

solar cell solutions with nanostructured surface are another example. Material properties and wave-matter interaction change near infrared and optical wavelengths in a way that does not allow direct translation of established design concepts to these frequencies. The special issue opens with an invited paper by Alù $e t a l$. that presents an in depth review of some of the concepts of optical antenna theory, design and applications. In another paper, Brockett et al. present a detailed electromagnetic characterization of GaAs photovoltaic nanopillar array solar cells for solar energy harvesting. The numerical results show significant increase in solar power absorbance over that of conventional bulk solar cells.

Phase shifters are an essential device in a phased array to allow for beam steering. At $\mathrm{mm}$ and sub $\mathrm{mm}$-waves those devices are expensive, complex and sometimes unreliable. Chen et al. present the conceptual vision of phase shifters based on integrally-gated graphene parallel-plate waveguides for operation at $\mathrm{THz}$ and infra-red. With this type of technology it might be possible in the future to realize low-loss and extremely compact on-chip programmable phasing devices.

Enhancement of $\mathrm{THz}$ wave generation efficiency is another continuous challenge. Khiabani et al. analyze the factors affecting the radiated power and optical-THz power conversion efficiency of a $\mathrm{THz}$ photoconductive antenna, using an equivalent circuit lumped-elements model.

Space borne calibration targets are currently a common practice for in-flight passive radiometer calibration for remote sensing applications. Measurement reliability depends on the accuracy of the load, which is influenced by its reflectivity level across the band and temperature uniformity over the device. Design constraints are its mass and volume. Murk et al. present a new load configuration that offers a good compromise between radiometric performance and the design constrains. Radiometric performance is evaluated by simulation and measurements on a breadboard model. Thermal behavior of the structure is studied by simulation.

\section{B. Compact Antennas and Antennas in Package (12 Papers)}

There is an increasing demand for low-cost wireless communication and imaging systems that operate in the mm-wave bands. Recent advances in silicon technologies have enabled single chip solutions, resulting in substantial cost reductions. However, to flourish the market of these mm-wave systems, not only are low-cost radio frequency integrated circuit (RFIC) solutions required, but also low-cost RFIC packages with integrated antennas. A paper by Tsutsumi et al. presents an antenna in package solution for $60 \mathrm{GHz}$ in which a loop antenna consists of two bonding wires connecting to a complementary metal-oxide-semiconductor (CMOS) chip and a metal plate on an interposer in a ball grid array (BGA) package. The antenna can be fabricated at low cost adopting a conventional BGA package fabrication process. The paper by Beer et al. proposes a packaging solution for the integration of a monolithic microwave integrated circuit (MMIC) and a thin film antenna into a single surface-mountable package. As the antenna is placed above an air cavity, a large bandwidth is achieved for the antenna. An ultimate low cost V-band solution comes from a paper by Hong et al. In this paper, the antenna is implemented on the application board directly, thus eliminating a traditional antenna package and its complicated grid array assemblies.

Most antenna-in-package designs use planar structures, such as patch, dipole, loop or slot. The paper by Enayati et al. proposes a horn-type structure at $60 \mathrm{GHz}$. The design yields widebandwidth and is suitable for array applications where nonline-of-sight communication is needed or multi-path spatial diversity techniques are to be implemented. A bond-wire-free interconnection between a MMIC and an E-band antenna using a stacked patch configuration is investigated by Smith et al.A short horn has been used to increase the antenna directivity. Alternatively, Ohlsson et al. present a slot-coupled semiconductor dielectric resonator antenna designed for on-chip assembly. The antenna has an impedance bandwidth of $6.1 \%$ in the V-band and a radiation efficiency of $98 \%$.

There are many papers discussing on-chip antenna designs. On-chip antennas typically have either very low radiation efficiency or very narrow impedance bandwidth due to the $\mu \mathrm{m}$-range distance between the planar antenna structure and the ground plane in conventional complementary metal-oxide-semiconductor (CMOS) or SiGe processes and the lossy silicon used (resistivity typically in the $1-10 \Omega . c m$ range). The paper by Marnat et al. proposes a new movable plate concept to realize mm-wave vertical on-chip antennas through microelectromechanical systems (MEMS) based post-processing steps in a CMOS compatible process. By virtue of its vertical position, the antenna is isolated from the lossy Si substrate and hence performs with a better efficiency as compared to the horizontal position. In addition, the movable plate concept enables polarization diversity by providing both horizontal and vertical polarizations on the same chip.

Most of the proposed $60 \mathrm{GHz}$ antennas in package present a boresight radiation pattern. However for some applications it is actually desirable to have an end-fire antenna. A wide-angle radiation pattern fan-like printed antenna designed for wireless communications in the V-band is proposed by Sun et al. 
A different application scenario is addressed in the article by Yeh et al., namely chip-to-chip communications. Wireless communications in principle can reduce the crowdedness and even improve system performance associated with wired connections. Comparing to a regular patch antenna, the patch antenna with an artificial magnetic conductor layer provides not only gain improvement in the horizontal direction but also very wide impedance bandwidth.

Other compact but non-packaged antenna solutions are also included in this section. Developing wideband architectures with multiple-input-multiple-output (MIMO) antenna systems at mm-wave offer many advantages including jointly optimized analogue and digital signal processing. This allows for flexible antenna designs and insertion loss reduction, as many passive structures can be avoided at both sides of the link. The paper by Ariza et al. presents a $60 \mathrm{GHz}$ polarimetric MIMO sensing system architecture that includes analogue miniaturized front ends. This architecture permits compact MIMO radar designs, multi-dimensional channel sounding for multi-gigabit communications, and the study of indoor polarimetric wireless channels in static and shadowed conditions due to human activity. In another paper, Bhattacharyya et al. propose a new analysis method for ring-slot elements backed by ground plane via cavities performed using a hybrid matrix formulation. The validity of the method has been demonstrated against finite element method (FEM) simulations while the new formulation is at least one order of magnitude faster. An optically transparent $\mathrm{CPW}$-fed monopole lozenge antenna using silver grid layer is described by Hautcoeur et al. for $60 \mathrm{GHz}$ security applications.

\section{Focusing/Collimating Antennas (14 Papers)}

Antennas for $\mathrm{mm}$ and sub $\mathrm{mm}$-wave applications typically require high gain to compensate for high free-space propagation loss. Renewed interest is for indoor multi-gigabit links or for automotive road safety applications. At these frequencies lenses are an attractive solution either as a primary feed or as a standalone moderate gain antenna. Nguyen et al. present a shaped dome V-band dielectric lens with stable Gaussian-like radiation pattern over a $25 \mathrm{GHz}$ bandwidth. This configuration presents advantage of lower material losses and lower weight compared to conventional lens solutions. Dielectric lenses can be used as array elements for beam steering as proposed by Artemenko et $a l$. This requires fewer array elements than a phased array for the same gain and allows replacing expensive phase shifters by RF switches.

In some application like automotive radars, a narrow beam is required only in one plane while in the orthogonal plane the beam can be broader. In this case instead of a 3D lens, a flat lens can be used. Lafond et al. present a flat Luneburg lens with reconfigurable feeds using individual MMIC amplifier and single pole, single throw (SPST) switch at each feed branch for very wide-angle electronic beam scanning. The paper demonstrates the feasibility of the concept at the $60 \mathrm{GHz}$ band.

Fresnel zone plates (FZP) can be used as an alternative to 3D dielectric lenses when large bandwidths are not required. The FZP antennas feature the merits of planar surface, reduced height and weight, when compared with the conventional dielectric lenses. A compact cavity-backed FZP lens antenna is presented by $\mathrm{Xu}$ et al. The antenna presents a boresight gain of $20.8 \mathrm{dBi}$ and a $3 \mathrm{~dB}$ gain bandwidth from 266 to $275 \mathrm{GHz}$. The back cavity improves aperture illumination, suppresses back radiation and reduces spillover losses. In a similar topic, Gallacher et al. introduce the design and optimization of a photo-injected FZP antenna. In this type of antennas the transmission loss of the different confined regions on the FZP plasma can be optically controlled. This way it is possible to dynamically focus and/or steer a directive beam at $\mathrm{mm}$ - or sub $\mathrm{mm}$-waves.

Reflector antennas may be more favorable when much higher gains are required. Unlike lenses, the aperture size can be increased without increase of losses. A short bowtie antenna with a broadband impedance bandwidth from 56 to $100 \mathrm{GHz}$ is presented by Qu et al. The gain is increased up to $18.5 \mathrm{dBi}$ by placing the bowtie in the focus of a small elliptically shaped metallic cavity.

Reflectarray is a type of reflector than can be made reconfigurable by dynamic altering the properties of each unit-cell. Compared to conventional phased arrays, reconfigurable reflectarrays tend to have lower cost and ease of construction. The paper by Perez-Palomino et al. presents a more flexible reconfigurable reflectarray concept obtained by printing it above a liquid crystal. The model takes into account the anisotropy and inhomogeneity of the liquid crystal. A demonstrating prototype is designed and measured in the F-band.

A particular challenge for the design of a reflector system at $\mathrm{mm}$ - and sub mm-waves is the design of feasible feeding elements. The methodology and tools for the design of short, ultra-low side-lobe corrugated feed horns in the $\mathrm{mm}$ - and the sub mm-wave bands is presented by McKay et al. Furthermore, the challenge increases when high density of feeds is required in the reflector focal plane to improve spatial information, as happens with imaging applications. Renker et al. analyze the focal plane array for a Stratosphere-Troposphere radiometer, while Trichopoulos et al.introduce a focal plane array sensor for $\mathrm{THz}$ imaging in the $0.6-1.2 \mathrm{THz}$ band. Each pixel in the sensor array consists of broadband $\mathrm{THz}$ antennas monolithically integrated with ultra-fast heterostructure backward diodes for $\mathrm{THz}$ sensing.

Sun et al. present a gain enhancement method for an antipodal tapered slot antenna by using zero-index metamaterial in the open aperture region of the antenna. Adding the zero-index metamaterial does not affect the antenna impedance much and allows a gain of 10.6-13.1 dBi over $60 \mathrm{GHz}$ band. The paper by Ghassemi and $\mathrm{Wu}$ describes another gain enhancement method by dielectric-loading the antenna in the aperture area. Compared to the zero-index metamaterial method, this one provides more gain improvement and maintains a much wider bandwidth. In a similar topic, Taringou et al. present a long broadband antipodal tapered slot antenna fed by a coplanar waveguide (CPW) instead of the more common microstrip feeding. CPW technology has some advantage over microstrip lines which include surface-mount integration, lower phase velocity variation, lower cross talk and radiation. 
Determination of the far-field radiation pattern of a high gain antenna using the classical near-filed to far-field transformation at $\mathrm{mm}$ - and sub mm-waves may be hindered by the difficulties to accurately retrieve phase. Junkin describes a new technique for accurate phase retrieval from the far-fields radiated by a Terahertz antenna from measured near-field intensity. Authors use graphics processing units (GPUs) to obtain almost real-time accurate far-field calculations.

\section{Arrays and Array Theory (8 Papers)}

Arrays are an alternative to focusing antennas when high gain is required but low profile is mandatory, for example, to allow package level integration. Due to its high manufacturing precision and capabilities of implementing complicated via structures, low temperature co-fired ceramics (LTCC) technology is a promising candidate for mm-wave antenna arrays. One major issue with mm-wave planar arrays is the relative strong surface wave due to large substrate size, particularly with high dielectric constant LTCC substrates. There are two major methods to reduce surface waves. One is to use low dielectric substrate such as foam materials or air cavity in the design. But air cavity increases manufacturing difficulties and cost while foam is very limited for mm-wave applications. The other method is to use electromagnetic band-gap (EBG) or ring structures around the antenna radiator to reduce surface waves. But it is difficult to use these concepts in array designs. The paper by Wang et al. proposes to use soft-surface structures comprised of metal strips and via fences to improve the radiation performance by reducing the losses due to severe surface waves and mutual coupling between adjacent elements. She et al. describe an oversized rectangular waveguide slot array antenna in V-band with an air layer in the radiating part to enhance the antenna gain. The array is fabricated using LTCC. The insertion of the air layer allows for a reduction on dielectric loss and bandwidth enhancement. In a similar topic, $X u$ et al. present a broadband and high gain array operating at $140 \mathrm{GHz}$ using dielectric-loaded slots fed by a substrate integrated waveguide (SIW). The configuration is electrically robust and has a simple design for fabrication using LTCC technology. In the $270 \mathrm{GHz}$ band, the previous authors now introduce a planar high gain linearly polarized radial line slot array antenna manufactured in LTCC technology.

Sano et al. present a linear slot array in a hollow rectangular coaxial waveguide in the V-band. This configuration allows fabrication using a different technology: diffusion bonding of laminated thin metal plates. A particularly interesting solution is presented to support the inner conductor in the center of the hollow waveguide. A totally different array technology and support material is discussed in Chahat et al. paper, It demonstrates a microstrip patch antenna array printed on commercial textiles at mm-waves for wearable applications and proposes a new fabrication process for reliable and accurate manufacturing of mm-wave microstrip antennas on textiles.

This section includes also new array concept developments. A possible array configuration to obviate the need for precise phase shifter is presented by Krieger et al. The proposed architecture uses arrays with elements spaced more closely than otherwise required over the target aperture in conjunction with novel spatial-domain delta-sigma processing to enable high performance with low-resolution phase shifters.

A two-dimensional array is presented by Lemaitre-Auger et $a l$. as a solution to generate a collimated and confined Bessel beam. The authors investigate both the array lattice and the effect of sub-sampling the array in order to reduce the number of antenna elements.

\section{E. Channel Modeling and Characterization (4 Papers)}

Indoor and outdoor wireless fixed and mobile communication services are steadily demanding ever increasing capacity to support huge traffic growth generated by increasing number of users and increasing number of mobile user broadband platforms and applications. Required bandwidth for next generation cellular networks is available at the under-utilized millimeter-wave spectrum. Adequate channel characterization is required at these frequencies in the context of cellular communications, using high gain beam-steerable antennas. Rappaport et al. present an experimental study on mm-wave propagation for outdoor cellular communication systems in urban environment, considering high gain beam steering antennas. The paper presents measurement results for the $40 \mathrm{GHz}$ band and propagation models relevant for future planning and deployment of such mobile systems. With a similar motivation, García-Rubia et al. analyze the results from a two-year rain attenuation measurement campaign at $80 \mathrm{GHz}$ over a horizontal $840 \mathrm{~m}$ link at Madrid. Measured attenuation results are compared with established theoretical models using drop-size distribution functions obtained from parallel experiments carried out at the same location.

Multi gigabit networks are being studied for indoor scenarios at millimeter waves. As for outdoor scenarios, high gain antennas are required to ensure the link budget, but obstruction by the human body may impair the service. Takahashi et al. propose a solution to minimize communication interruption at 60 $\mathrm{GHz}$ band by the human body by using artificial multi reflectors. The improvements by the proposed method are quantified; the strategy is generally applicable to most indoor communications environments.

Very high data-rates are also required for emerging body-area network applications. Again millimeter-waves are a potential candidate to accommodate the required large bandwidths and so channel model characterization is needed for this very specific type of scenarios. Chahat et al. characterize the electromagnetic fields propagating at the skin/air interface at $60 \mathrm{GHz}$ for on-body networks. The study comprises analytical formulation, full-wave simulation and experiments. The main focus is on the interpretation, in the near-skin context, of the different terms from the old Norton approximate expressions derived from Sommerfled's formulation for a dipole near a lossy medium. The interest of the paper is put also on the determination of the power loss exponent in different regions.

\section{ACKNOWLEDGMENT}

The Guest Editors want to express their sincere thanks to Prof. Michael A. Jensen, Editor-in-Chief of the IEEE TRANSACTIONS ON ANTENNAS AND PROPAGATION, for supporting, encouraging 
and overseeing in the elaboration [I think you mean "collaboration" and not "elaboration"] and publication of this special issue. They thank all the authors for their outstanding contributions and the more than 200 reviewers who were involved in the reviewing process of more than 90 submissions. As we all know, the quality of the published articles highly depends on the authors and also on the work done by the reviewers with their useful suggestions and comments that helped improving the overall quality of the papers. To all, thank you.

\section{Carlos Fernandes Fernandes, Guest Editor}

Instituto de Telecomunicações, Instituto Superior Técnico

Technical University of Lisbon

Lisbon, Portugal
DuIXIAN LiULiU, Guest Editor

IBM T. J. Watson Research Centre

Yorktown Heights, NY USA

Jiro HirokawaHirokawa, Guest Editor

Tokyo Institute of Technology

Tokyo, Japan

Jorge CostaCosta, Guest Editor

Instituto de Telecomunicações

University Institute of Lisbon (ISCTE-IUL)

Lisbon, Portugal

Ronan SauleauSauleau, Guest Editor

University of Rennes 1

Rennes, France

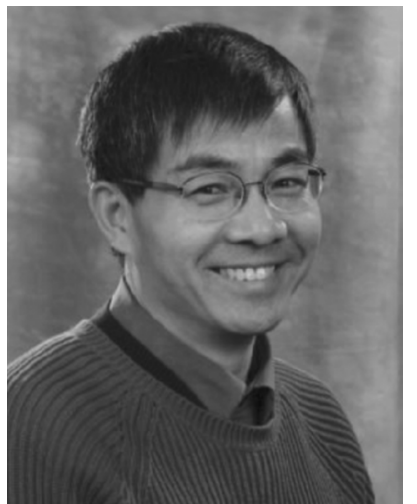

Duixian Liu (S'85-M'90-SM'98-F'10) received the B.S. degree in electrical engineering from XiDian University, Xi'an, China, in 1982, and the M.S. and Ph.D. degrees in electrical engineering from the Ohio State University, Columbus, OH, USA, in 1986 and 1990, respectively.

From 1990 to 1996, he was with Valor Enterprises Inc., Piqua, OH, USA, initially as an Electrical Engineer and then as the Chief Engineer, during which time he designed an antenna product line ranging from $3 \mathrm{MHz}$ to $2.4 \mathrm{GHz}$ for the company, a very important factor for the prestigious Presidential "E" Award for Excellence in Exporting in 1994. Since April 1996, he has been with the IBM T. J. Watson Research Center, Yorktown Heights, NY, USA, as a Research Staff Member. He has received three IBM's Outstanding Technical Achievement Awards and one Corporate Award, the IBM's highest technical award. He was named Master Inventor in 2007. He has co-edited a book titled Advanced Millimeter-wave Technologies-Antennas, Packaging and Circuits (Wiley, 2009). He has authored or coauthored more than 100 journal and conference papers.

Dr. Liu is a Fellow of IEEE. He received the Best Paper Prize of the 2007 IEEE International Workshop on Antenna technology for “Antenna-in-package in LTCC for 60 GHz Radio," IBM Research's 2011 Pat Goldberg Memorial Best Paper Award in Computer Science, Electrical Engineering and Math for "Organic Packages with Embedded PhasedArray Antennas for 60-GHz Wireless Chipsets," and the 2012 S. A. Schelkunoff Prize Paper Award of the IEEE Antennas and Propagation Society for "Dual Grid Array Antennas in a Thin-Profile Package for Flip-Chip Interconnection to Highly Integrated 60-GHz Radios," He has 40 patents issued and 20 patents pending. His research interests are antenna design, EM modeling, chip packaging, digital signal processing, and communications technologies. He is an Associate Editor for the IEEE TRANSACTIONS ON Antennas And Propagation and was a Guest Editor for the July 2009 IEEE Transactions ON ANTENNAS AND PROPAGATION Special Issue on Antennas and Propagation Aspects of 60-90 GHz Wireless Communications. He has been the Organizer or Chair for numerous international conference sessions or special sessions and also a technical program committee member. He was the General Chair of the 2006 IEEE International Workshop on Antenna Technology: Small Antennas and Novel Metamaterials, White Plains, NY. He has served as an external Ph.D. examiner for several universities and external examiner for government organizations on research grants.

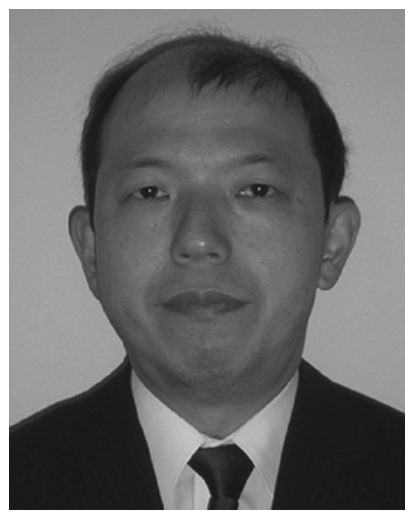

Jiro Hirokawa (S'89-M'90-SM'03-F'12) was born in Tokyo, Japan, on May 8, 1965. He received the B.S., M.S., and D.E. degrees in electrical and electronic engineering from Tokyo Institute of Technology (Tokyo Tech), Tokyo, Japan in 1988, 1990 and 1994, respectively.

He was a Research Associate from 1990 to 1996, and is currently an Associate Professor at Tokyo Tech. From 1994 to 1995, he was with the antenna group of Chalmers University of Technology, Gothenburg, Sweden, as a Postdoctoral Fellow. His research area has been in slotted waveguide array antennas and millimeter-wave antennas.

Prof. Hirokawa is an IEEE Fellow and a Senior Member of IEICE. He received an IEEE AP-S Tokyo Chapter Young Engineer Award in 1991, a Young Engineer Award from IEICE in 1996, a Tokyo Tech Award for Challenging Research in 2003, a Young Scientists' Prize from the Minister of Education, Cultures, Sports, Science and Technology in Japan in 2005, a Best Paper Award in 2007, a Best Letter Award in 2009 from IEICE Communications Society and Asia Pacific Microwave Conference prize in 2011 and 2012. 

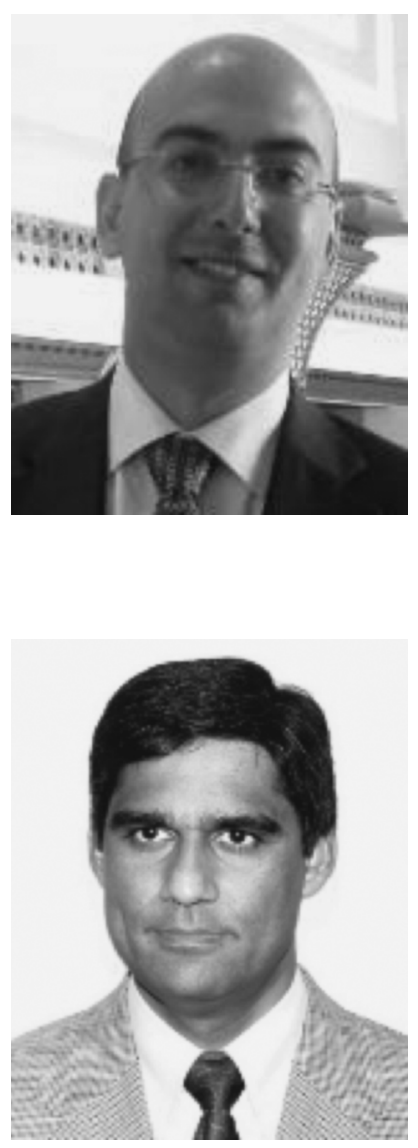

and metamaterials.

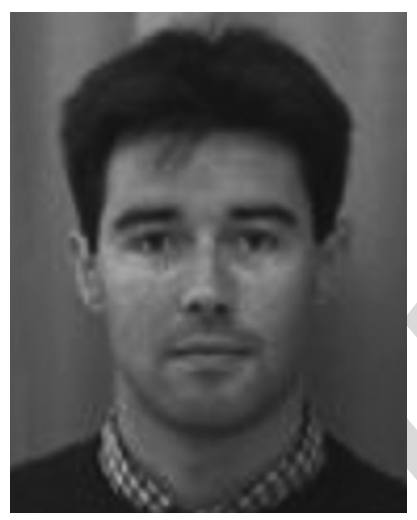

Jorge R. Costa (S'97-M'03-SM'09) was born in Lisbon, Portugal, in 1974. He received the Licenciado and $\mathrm{Ph} . \mathrm{D}$. degrees in electrical and computer engineering from the Instituto Superior Técnico (IST), Technical University of Lisbon, Lisbon, Portugal, in 1997 and 2002, respectively.

He is currently a Researcher at the Instituto de Telecomunicações, Lisbon, Portugal. He is also an Associate Professor at the Departamento de Ciências e Tecnologias da Informação, Instituto Universitário de Lisboa (ISCTE-IUL). His present research interests include lenses, reconfigurable antennas, MEMS switches, UWB, MIMO and RFID antennas. He is the coauthor of four patent applications and more than 50 contributions to peer reviewed journals and international conference proceedings. More than ten of these papers have appeared in IEEE Journals.

Prof. Costa is currently serving as an Associate Editor for the IEEE TRANSACTIONS ON Antennas and Propagation.

Carlos A. Fernandes (S'86-M'89-SM'08) received the Licenciado, M.Sc., and Ph.D. degrees in Electrical and computer engineering from the Instituto Superior Técnico (IST), Technical University of Lisbon, Lisbon, Portugal, in 1980, 1985, and 1990, respectively.

In 1980, he joined IST where, since 2006, he is a Full Professor in the Department of Electrical and Computer Engineering in the areas of microwaves, radio wave propagation and antennas. He is a Senior Researcher at the Instituto de Telecomunicações and member of the Board of Directors. He has been the leader of antenna activities in National and European Projects as RACE 2067-MBS (Mobile Broadband System), ACTS AC230 - SAMBA (System for Advanced Mobile Broadband Applications) and ESA/ESTEC-ILASH (Integrated Lens Antenna Shaping). He has coauthored a book, a book chapter, and more than 130 technical papers in peer reviewed international journals and conference proceedings, in the areas of antennas and radiowave propagation modeling. Current research interests include dielectric antennas for millimeter wave applications, antennas and propagation modeling for personal communication systems, RFID antennas, artificial dielectrics

Ronan Sauleau (M'04-SM'06) received the B.Sc. degree in electrical engineering and radio communications from the Institut National des Sciences Appliquées, Rennes, France, in 1995. He received the Agrégation degree from the Ecole Normale Supérieure de Cachan, Cachan, France, in 1996, and the Ph.D. degree in signal processing and telecommunications and the "Habilitation à Diriger des Recherche" degree from the University of Rennes 1, Rennes, France, in 1999 and 2005 , respectively.

He was an Assistant Professor and Associate Professor at the University of Rennes 1, Rennes, France between September 2000 and November 2005, and between December 2005 and October 2009, respectively. He has been appointed as a Full Professor in the same University since November 2009. His current research fields are numerical modeling (mainly FDTD), millimeter-wave printed and reconfigurable (MEMS) antennas, substrate integrated waveguide antennas, lens-based focusing devices, periodic and nonperiodic structures (electromagnetic bandgap materials, metamaterials, reflectarrays, and transmitarrays), and biological effects of millimeter waves. He has been involved in more than 30 research projects at the national and European levels and has co-supervised 14 Postdoctoral Fellows, 24 Ph.D. students, and 40 Master students. He has received eight patents and is the author or coauthor of 135 journal papers and more than 285 publications in international conferences and workshops. He has shared the responsibility of the research activities on antennas at IETR in 2010 and 2011. He is now co-responsible for the research Department "Antenna and Microwave Devices" at IETR and is deputy director of IETR.

Prof. Sauleau received the 2004 ISAP Conference Young Researcher Scientist Fellowship (Japan) and the first Young Researcher Prize in Brittany, France, in 2001, for his research work on gain-enhanced Fabry-Perot antennas. In September 2007, he was elevated to junior member of the "Institut Universitaire de France". He was awarded the Bronze medal by CNRS in 2008. He was the co-recipient of several international conference awards (Int. Sch. of BioEM 2005, BEMS'2006, MRRS'2008, E-MRS'2011, BEMS'2011, IMS'2012, Antem'2012). 